\title{
Improved BDFE Using A Priori Information for Turbo Equalization
}

\author{
Jingxian Wu, Member, IEEE, Sang-Yick Leong, Kah-Ping Lee, \\ Chengshan Xiao, Senior Member, IEEE, and Jan C. Olivier
}

\begin{abstract}
Turbo equalization improves communication system performance by iteratively exchanging information between soft-input soft-output (SISO) equalizer and SISO channel decoder. The trellis-based maximum a posteriori probability (MAP) algorithm serves as the optimum SISO equalizer for turbo equalization. However, MAP algorithm is unsuitable for systems with large modulation constellation size and severe inter-symbol interference (ISI) due to its prohibitively high computational complexity. In this paper, an improved SISO block decision feedback equalizer (BDFE) is proposed for low complexity turbo equalization. Unlike other sub-optimum equalizers which perform symbol by symbol detection, the proposed equalizer generates the soft output for each data bit by collecting information from a sequence of samples as in MAP algorithm. The sequencebased equalization is enabled by using not only soft a priori input from channel decoder, but also hard a priori information obtained from BDFE in previous iteration. The combination of soft $a$ priori information and hard $a$ priori information renders better performance with less iterations compared to other suboptimum algorithms. In addition, the computational complexity of the proposed algorithm is on the same order as conventional SISO BDFE algorithm, and is much lower compared to the trellis-based MAP algorithm.
\end{abstract}

Index Terms-Block decision feedback equalization, iterative equalization, sequence detection, turbo equalization.

\section{INTRODUCTION}

$\mathbf{T}$ URBO equalization is a powerful iterative receiver that can be employed by systems operating in an inter-symbol interference (ISI) environment, with data protected by channel coding and interleaver. Receiver with turbo equalization treats the channel code and the ISI channel as the concatenation of two trellis-based structures separated by an interleaver. The performance of the system is improved by iteratively exchanging extrinsic information between a soft-input softoutput (SISO) equalizer and an SISO channel decoder until convergence is achieved.

Manuscript received July 11, 2006; revised January 28, 2007; accepted April 9, 2007. The editor coordinating the review of this paper and approving it for publication is Z. Tian. The work of C. Xiao was supported in part by the National Science Foundation under Grant CCF-0514770 and the Office of Naval Research under Grant N00014-07-1-0219. Part of this paper was previously presented at the 2004 IEEE Vehicular Technology Conference (VTC'04-Fall), Los Angles, CA.

$\mathrm{J}$. Wu is with the Department of Engineering Science, Sonoma State University, Rohnert Park, CA 94928 USA (e-mail: jingxian.wu@ sonoma.edu).

S. Y. Leong, K. P. Lee, and C. Xiao were with the University of MissouriColumbia, Columbia, MO, USA. C. Xiao is now with the Department of Electrical and Computer Engineering, University of Missouri-Rolla, Rolla, MO 65409 USA (e-mail: xiaoc@umr.edu).

J. C. Olivier is with the Department of Electrical, Electronic and Computer Engineering, University of Pretoria, RSA (e-mail: corne.olivier@eng.up.ac.za).

Digital Object Identifier 10.1109/TWC.2008.060463.
The concept of turbo equalizer was first proposed in [1], where soft-output Viterbi algorithm (SOVA) with maximum likelihood sequence estimation (MLSE) [2] - [4] is used for the calculation of extrinsic information in both SISO equalizer and SISO channel decoder. Replacing SOVA with maximum a posteriori probability (MAP) algorithm [5] - [6] leads to similar performance. SOVA algorithm or MAP algorithm can generate the best possible performance under the structure of turbo equalization. On the other hand, the computational complexity of MAP and SOVA algorithms grow exponentially with modulation constellation size $M$ and equivalent discrete-time channel length $L$. Large values of $M$ and $L$, which are common in modern communication systems, make equalization with MAP or SOVA algorithms intractable. As a consequence, a sub-optimum, reduced complexity, SISO equalizer with minimum performance degradation is highly desirable for efficient turbo equalization in practical systems.

The design of low complexity SISO equalizer has attracted considerable attentions recently [7] - [18]. In [7], a linear SISO minimum mean square error (MMSE) based equalizer is employed for the cancellation of both ISI and multiple access interference in a code division multiple access (CDMA) system. The linear MMSE weight vectors are derived by using the first order statistics (mean) calculated from the soft input. The method is extended in [8] - [10], where both first order statistics and second order statistics collected from soft input are used for the formulation of the linear MMSE equalizer. A non-linear, SISO block decision feedback equalizer (BDFE) is proposed in [11], where hard decisions from decision feedback is used for ISI cancellation. These algorithms can achieve a reasonable performance at a computational complexity much lower compared to the optimum MAP or SOVA algorithms.

In this paper, an improved SISO BDFE algorithm is proposed for turbo equalization. The new equalizer is motivated by the fact that all the low complexity equalizers mentioned above perform equalization on a symbol by symbol basis. The symbol by symbol detection is the key for complexity reduction, whereas it is also the main reason of performance degradation compared to sequence-based optimum equalizers. In the improved SISO BDFE algorithm, sequence-based detection is adopted, i.e., the statistics of each data bit is calculated by collecting information from a sequence of samples as in MAP algorithm. This sequence detection is made possible by employing not only soft a priori information from channel decoder, but also hard a priori information from previous iterations. The sub-optimum sequence-based estimation in the new method effectively mitigates the problem of error propagation suffered by conventional BDFE systems. 


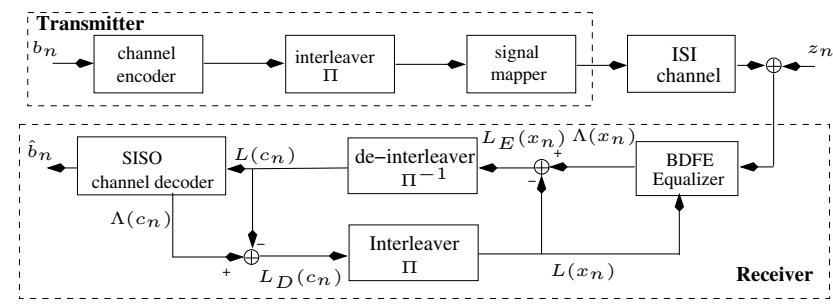

Fig. 1. Turbo equalization system model consists of SISO equalizer and channel decoder.

Both theoretical analysis and simulation results show that the improved SISO BDFE algorithm outperforms conventional SISO BDFE algorithm without sacrificing the computational complexity. In addition, due to the combination of soft a priori information and hard a priori information, the proposed algorithm converges much faster compared to algorithms which use soft a priori information only.

The rest of the paper is organized as follows. Section II introduces the system model and the principles of turbo equalization. Section III derives the improved SISO BDFE algorithm, and the complexity of the new algorithm is analyzed and compared to other systems. Simulation results are presented in Section IV, and Section V concludes the paper.

\section{System Model}

\section{A. Equivalent Discrete Time System Representation}

We consider the system model shown in Fig. 1. Prior to transmission, a sequence of binary information $b_{n} \in\{0,1\}$ is passed through a convolutional encoder followed by a block interleaver. The output of the interleaver is divided into blocks with length $K \cdot N$, where $K=\log _{2} M$ with $M$ being the modulation level, and $N$ is the number of modulated symbols in each block. Denote the binary block at the output of the interleaver as $\mathbf{x} \triangleq\left[\mathbf{x}_{1}, \mathbf{x}_{2}, \cdots, \mathbf{x}_{N}\right]^{T} \in \mathcal{B}^{K N \times 1}$, where $\mathbf{x}_{n} \triangleq$ $\left[x_{n, 1}, \cdots, x_{n, K}\right] \in \mathcal{B}^{1 \times K}, \mathbf{A}^{T}$ is the transpose of matrix $\mathbf{A}$, and $\mathcal{B}=\{-1,+1\}$. The output of the interleaver is modulated by mapping $\mathbf{x}_{n}$ to the $M$-ary modulation symbol $s_{n} \in \mathcal{S}$, where $\mathcal{S}$ is the modulation constellation set with cardinality $M=2^{K}$.

The modulated data sequence, $\mathbf{s}=\left[s_{1}, s_{2}, \cdots, s_{N}\right]^{T} \in$ $\mathcal{S}^{N \times 1}$, is transmitted in burst mode. The transmitted data is distorted by frequency selective fading and additive white Gaussian noise (AWGN). The discrete-time representation of the system is [19]

$$
y_{n}=\sum_{l=0}^{L-1} h_{l} s_{n-l}+z_{n}, \text { for } 1 \leq n \leq N,
$$

where $s_{n}$ is the modulated information symbol with average energy $E_{s}, y_{n}$ is the symbol rate sample at the output of the receive filter, $z_{n}$ is the zero-mean AWGN sample, and $h_{l}$ is the discrete-time composite impulse response (CIR) resulted from the cascade of the transmit filter, frequency selective fading, and receive filter [19]. The CIR is assumed to be quasi-static, i.e., it's constant within one block, but varies from block to block.

The system model described in (1) can be represented in matrix format as

$$
\mathbf{y}=\mathbf{H} \mathbf{s}+\mathbf{z}
$$

where $\mathbf{y}=\left[\begin{array}{llll}y_{1}, & y_{2}, & \cdots, & y_{N}\end{array}\right]^{T} \underset{\mathcal{C}_{N \times 1}}{\in} \mathcal{C}^{N \times 1}$, $\mathbf{s}=\left[\begin{array}{llll}s_{1}, & s_{2}, & \cdots, & s_{N}\end{array}\right]^{T} \in \mathcal{C}^{N \times 1}, \quad \mathbf{z}=$ $\left[z_{1}, z_{2}, \cdots, z_{N}\right]^{T} \in \mathcal{C}^{N \times 1}$ are the receive sample vector, modulation symbol vector, and noise vector, respectively, and the channel matrix $\mathbf{H}$ is defined as

$$
\mathbf{H}=\left(\begin{array}{cccccc}
h_{0} & 0 & 0 & \cdots & \cdots & 0 \\
\vdots & \ddots & & \ddots & & \vdots \\
0 & h_{L-1} & \cdots & h_{0} & \cdots & 0 \\
\vdots & & \ddots & & \ddots & \vdots \\
0 & \cdots & 0 & h_{L-1} & \cdots & h_{0}
\end{array}\right) \in \mathbf{C}^{N \times N}
$$

Decision feedback equalization requires that the channel impulse response be in its minimum phase form, i.e., the power of the channel impulse response concentrates on the leading taps, to avoid numerical instability. In some channel profiles, such as Typical Urban (TU) and Hilly Terrain (HT) of the GSM/EDGE system [20], the channel impulse response is not in its minimum phase form. Therefore the system needs to be transformed to an equivalent minimum phase system before equalization.

In $\mathrm{BDFE}$, the minimum phase system that is equivalent to (2) can be represented by [21]

$$
\mathbf{r}=\mathbf{W} \mathbf{y}=\mathbf{G s}+\mathbf{v},
$$

where $\mathbf{r}=\left[r_{1}, r_{1}, \cdots, r_{N}\right]^{T}, \mathbf{v}=\left[v_{1}, v_{2}, \cdots, v_{N}\right]^{T}$ are the receiver samples and noise samples of the equivalent system, respectively. The feedforward matrix, $\mathbf{W} \in \mathcal{C}^{N \times N}$, and feedback matrix, $\mathbf{G} \in \mathcal{C}^{N \times N}$, are solved by using the minimum mean square error (MMSE) criterion, and the solutions are [21]

$$
\begin{aligned}
\mathbf{G} & =\mathbf{U}, \\
\mathbf{W} & =\mathbf{U} \cdot \mathbf{H}^{H} \cdot\left(E_{s} \mathbf{H} \mathbf{H}^{H}+N_{0} \mathbf{I}_{N}\right)^{-1},
\end{aligned}
$$

where $\mathbf{I}_{N}$ is an $N \times N$ identity matrix, $\mathbf{A}^{H}$ stands for matrix Hermitian, and $\mathbf{U} \in \mathcal{C}^{N \times N}$ is obtained from the Cholesky decomposition of the matrix $\boldsymbol{\Phi}=\frac{1}{E_{s}} \mathbf{I}_{N}+\frac{1}{N_{0}} \mathbf{H} \mathbf{H}^{H}$ as $\boldsymbol{\Phi}=$ $\mathbf{U}^{H} \mathbf{D} \mathbf{U}$. The matrix $\mathbf{U}$ is an upper triangular matrix with unit diagonal elements, and $\mathbf{D} \in \mathcal{R}^{N \times N}$ is a diagonal matrix. The matrix $\mathbf{D}^{-1}$ is the covariance matrix of the noise components $\mathbf{v}$. Since $\mathbf{D}$ is diagonal, the elements of the noise vector, $\mathbf{v}$, are uncorrelated. The noise components can be approximated as a complex Gaussian distributed vector, i.e., $\mathbf{v} \sim \mathcal{N}\left(\mathbf{0}, \mathbf{D}^{-1}\right)$.

Due to the fact that $\mathbf{G}$ is upper triangular, we have $g_{n m}=$ $0, \forall n<m$. Therefore the information symbols are detected in reverse order, i.e., $s_{N}$ is detected first and $s_{1}$ is detected last within one block. The hard output decision feedback equalization can be described as

$$
\begin{aligned}
\hat{s}_{N}= & \underset{s_{N} \in \mathcal{S}}{\operatorname{argmin}}\left|r_{N}-g_{N, N} s_{N}\right|^{2}, \\
\hat{s}_{n}= & \underset{s_{n} \in \mathcal{S}}{\operatorname{argmin}}\left|r_{n}-g_{n, n} s_{n}-\sum_{m=n+1}^{N} g_{n, m} \hat{s}_{m}\right|^{2}, \\
& \quad \text { for } n=N-1, \cdots, 1,
\end{aligned}
$$

where $g_{n, m}$ is the element on the $n$th row and $m$ th column of $\mathbf{G}$, and $\hat{s}_{m}$, for $m=n+1, \cdots, N$, are the hard decision symbols from feedback. 


\section{B. Principle of Turbo Equalization}

Turbo equalization utilizes the iterative exchange of soft information between equalizer and channel decoder to improve system performance. The block diagram of a turbo equalizer can be found at the receiver portion of Fig. 1. Each iteration of Turbo equalization consists of two stages, an SISO equalizer followed by an SISO channel decoder. The equalizer and channel decoder are separated by an interleaver, $\Pi(\cdot)$, and a de-interleaver, $\Pi^{-1}(\cdot)$.

During each iteration, the equalizer calculates the a posteriori $\log$ likelihood ratios (LLR) for each information bit $x_{n}$ as

$$
\Lambda\left(x_{n} \mid \mathbf{r}\right)=\ln \frac{P\left(x_{n}=+1 \mid \mathbf{r}\right)}{P\left(x_{n}=-1 \mid \mathbf{r}\right)} .
$$

Using Bayes' Rule, we can write (9) as

$$
\begin{aligned}
\Lambda\left(x_{n} \mid \mathbf{r}\right) & =\ln \frac{\sum_{\forall \mathbf{x}: x_{n}=+1} P(\mathbf{r} \mid \mathbf{x}) P(\mathbf{x})}{\sum_{\forall \mathbf{x}: x_{n}=-1} P(\mathbf{r} \mid \mathbf{x}) P(\mathbf{x})} \\
& =\underbrace{\ln \frac{\sum_{\forall \mathbf{x}: x_{n}=+1} P(\mathbf{r} \mid \mathbf{x}) \prod_{n^{\prime} \neq n} P\left(x_{n^{\prime}}\right)}{\sum_{\forall \mathbf{x}: x_{n}=-1} P(\mathbf{r} \mid \mathbf{x}) \prod_{n^{\prime} \neq n} P\left(x_{n^{\prime}}\right)}}_{L_{E}\left(x_{n}\right)}+L\left(x_{n}\right) .
\end{aligned}
$$

where $L\left(x_{n}\right) \triangleq \ln \frac{P\left(x_{n}=+1\right)}{P\left(x_{n}=-1\right)}$ is the a priori information of the information bit $x_{n}$. In Turbo equalization, the a priori information, $L\left(x_{n}\right)$, is obtained by interleaving the soft-output of the channel decoder from the previous iteration. In the first iteration, there is no a priori information available and we have $L\left(x_{n}\right)=0, \forall n$.

The extrinsic LLR, $L_{E}\left(x_{n}\right)$, contains the information obtained by exploring the structure of the frequency selective channel fading. Therefore, $L_{E}\left(x_{n}\right)$ is the soft-output of the equalizer, and it will be de-interleved to $L\left(c_{n}\right)=$ $\Pi^{-1}\left(L_{E}\left(x_{n}\right)\right)$, which is then used as the a priori information, or, soft-input, by the channel decoder.

The convolutional decoder calculates the extrinsic LLR for each code bit by exploring the code trellis structure as well as the a priori information $L\left(c_{n}\right)$. The extrinsic LLR for the code bit, $c_{n}$, can be expressed as

$L_{D}\left(c_{n}\right)=\ln \frac{P\left(c_{n}=+1 \mid L\left(c_{1}\right), \cdots, L\left(c_{N_{c}}\right)\right)}{P\left(c_{n}=-1 \mid L\left(c_{1}\right), \cdots, L\left(c_{N_{c}}\right)\right)}-\underbrace{\ln \frac{P\left(c_{n}=+1\right)}{P\left(c_{n}=-1\right)}}_{L\left(c_{n}\right)}$.

where $N_{c}$ is the number of code bits in each block.

The extrinsic LLR at the output of the convolutional decoder is interleaved as $L\left(x_{n}\right)=\Pi\left(L_{D}\left(c_{n}\right)\right)$, which is fed into the equalizer as the a priori information for the next iteration. At the final iteration, the SISO convolutional decoder estimates the original binary information $b_{n} \in\{0,1\}$ as

$$
\hat{b}_{n}=\underset{b_{n} \in\{0,1\}}{\operatorname{argmax}} P\left(b_{n} \mid L\left(c_{1}\right), \cdots, L\left(c_{N_{c}}\right)\right) .
$$

It is important to note that the statistically independent a priori LLRs $L_{E}\left(x_{n}\right)$ and $L_{D}\left(c_{n}\right)$ are fed back to each other iteratively and lead to improvement in BER performance. This essential feature achieves the turbo principle. However, after the first iteration, $L_{E}\left(x_{n}\right)$ and $L_{D}\left(c_{N}\right)$ become more correlated through the iterations. As a consequence, the improvement will diminish after a large number of iterations and therefore a termination criterion is required to stop the iterative process.

In both the equalizer and convolutional decoder, the exact $a$ posteriori LLR can be calculated by using the optimum MAP algorithm or SOVA algorithm, which can effectively explore the trellis structure of the frequency selective channel or convolutional code, However, the complexity of the optimum equalizers increases exponentially with the modulation level $M$ and channel length $L$. The computational complexity makes it prohibitively expensive to implement the optimum MAP or SOVA algorithm in the equalizer. In the next section, a BDFE-based sub-optimum SISO equalization algorithm is presented to calculate an approximation of a posteriori LLR.

\section{Turbo Equalization Using BDFE}

An improved SISO BDFE channel equalization algorithm is presented in this section. The improved BDFE algorithm can be used in the channel equalizer for the calculation of the a posteriori LLR, with a much lower complexity compared to the optimum MAP algorithm.

\section{A. Conventional SISO BDFE Algorithm}

In this subsection, we review the SISO BDFE algorithm presented in [11], which motivates the improved BDFE algorithm proposed in this paper. For the sake of simplicity, BPSK modulation is used in this subsection. Higher level modulation schemes are discussed in the subsequent subsections.

With the operation of decision feedback, the a posteriori probability (APP) of the information symbol $s_{n}$ is calculated on a symbol by symbol base in [11] as

$$
P\left(s_{n} \mid r_{n}\right)=\frac{P\left(r_{n} \mid s_{n}, \hat{\mathbf{s}}_{n+1}\right) P\left(s_{n}\right)}{P\left(r_{n}\right)},
$$

where $\hat{\mathbf{s}}_{n+1}=\left[\hat{s}_{n+1}, \cdots, \hat{s}_{N}\right]$ are deterministic, hard detected symbols from decision feedback with $P\left(\hat{\mathbf{s}}_{n+1}\right)=1$, and

$$
\begin{array}{r}
P\left(r_{n} \mid s_{n}, \hat{\mathbf{s}}_{n+1}\right)= \\
\frac{1}{\pi \sigma_{n}^{2}} \exp \left(-\frac{1}{\sigma_{n}^{2}}\left|r_{n}-g_{n, n} s_{n}-\sum_{m=n+1}^{N} g_{n, m} \hat{s}_{m}\right|^{2}\right),
\end{array}
$$

where $\sigma_{n}^{2}=d_{n, n}^{-1}$, with $d_{n, n}$ being the $n$th diagonal element of the diagonal matrix $\mathbf{D}$. The Gaussian approximation of the noise vector $\mathbf{v}$ is used in (12).

Based on the APP defined in (11) and (12), the symbol by symbol a posteriori LLR is

$$
\begin{array}{r}
\Lambda\left(s_{n} \mid r_{n}\right)=\underbrace{\ln \frac{P\left(s_{n}=+1\right)}{P\left(s_{n}=-1\right)}}_{L\left(s_{n}\right)}+ \\
\underbrace{\ln \frac{\exp \left(-\frac{1}{\sigma_{n}^{2}}\left|r_{n}-g_{n, n}(+1)-\sum_{m=n+1}^{N} g_{n, m} \hat{s}_{m}\right|^{2}\right)}{\exp \left(-\frac{1}{\sigma_{n}^{2}}\left|r_{n}-g_{n, n}(-1)-\sum_{m=n+1}^{N} g_{n, m} \hat{s}_{m}\right|^{2}\right)}}_{L_{E}\left(s_{n}\right)} .
\end{array}
$$

The extrinsic information, $L_{E}\left(x_{n}\right)$, is used as the soft-output of the equalizer. In the mean time, hard decision of the $n$th 
information bit is made based on the LLR as

$$
\hat{s}_{n}=\underset{s_{n} \in\{-1,+1\}}{\operatorname{argmax}} \Lambda\left(s_{n} \mid r_{n}\right) .
$$

The hard decision $\hat{s}_{n}$ is fed back and used in the detection of $s_{m}$, for $m<n$.

It should be noted that the LLR defined in (13) is different from $\Lambda\left(x_{n} \mid \mathbf{r}\right)$ described in (9), where the APP of each information symbol is calculated by conditioning on a sequence of samples, r. The LLR in (13) is calculated by considering only one receive sample, $r_{n}$, and $N-n+1$ hard detected symbols, $\hat{\mathbf{s}}_{n+1}$. This sub-optimum calculation of the a posteriori LLR is due to the operation of BDFE, which performs detection on a symbol by symbol basis. As a result, the soft-output of the equalizer, $L_{E}\left(x_{n}\right)$, is only a rough approximate of the extrinsic information defined in (9). Therefore, the performance of this conventional SISO BDFE is usually unsatisfactory in the context of turbo equalization, especially for high level modulations with severe multipath channels.

\section{B. Improved SISO BDFE}

Here we proceed to an improved SISO BDFE algorithm which can generate a more accurate extrinsic information at the output of the equalizer.

As discussed in the previous subsection, the main limitation of the conventional SISO BDFE is the calculation of APP, which depends on only one received sample, $r_{n}$, instead of the entire sequence of samples, $\mathbf{r}$. Motivated by this fact, we define the APP of the $n$th modulation symbol, $s_{n} \in \mathcal{S}$, in the $i$ th iteration as

$$
P\left(s_{n}^{(i)} \mid \mathbf{r}\right)=\frac{P\left(\mathbf{r} \mid s_{n}\right) P\left(s_{n}\right)}{p(\mathbf{r})} .
$$

Since the sequence $\mathbf{r}$ depends on $\left[s_{1}, \cdots, s_{N}\right]$, the likelihood function, $P\left(\mathbf{r} \mid s_{n}\right)$, can be expressed as

$P\left(\mathbf{r} \mid s_{n}\right)=P\left(\mathbf{r} \mid s_{n}, \hat{s}_{1}^{(i-1)}, \cdots, \hat{s}_{n-1}^{(i-1)}, \hat{s}_{n+1}^{(i)}, \cdots, \hat{s}_{N}^{(i)}\right)$,

where $\left[\hat{s}_{n+1}^{(i)}, \cdots, \hat{s}_{N}^{(i)}\right]$ are hard decisions from the current iteration, and $\left[\hat{s}_{1}^{(i-1)}, \cdots, \hat{s}_{n-1}^{(i-1)}\right]$ are hard decisions from the previous iteration. The equation holds since all the hard decisions are deterministic.

Combining (15) and (16), we have

$$
P\left(s_{n}^{(i)} \mid \mathbf{r}\right)=\frac{P\left(\mathbf{r} \mid \mathbf{s}_{n}^{(i)}\right) P\left(s_{n}\right)}{p(\mathbf{r})} .
$$

where $\mathbf{s}_{n}^{(i)}=\left[s_{n}, \hat{s}_{1}^{(i-1)}, \cdots, \hat{s}_{n-1}^{(i-1)}, \hat{s}_{n+1}^{(i)}, \cdots, \hat{s}_{N}^{(i)}\right]$.

From (4) and the fact that the equivalent channel matrix, $\mathbf{G}$, is upper triangular, the conditional probability, $P\left(\mathbf{r} \mid \mathbf{s}_{n}^{(i)}\right)$, can be written as

$$
p\left(\mathbf{r} \mid \mathbf{s}_{n}^{(i)}\right)=\prod_{m=1}^{N} \frac{1}{\pi \sigma_{m}^{2}} \exp \left\{-\frac{\left|\rho_{n, m}\left(s_{n}\right)\right|^{2}}{\sigma_{m}^{2}}\right\},
$$

where the values of $\rho_{n, m}\left(s_{n}\right)$ is expressed in (19) at the top of the next page.
Following the a posteriori LLR definition given in (9), we can write the LLR, $\Lambda\left(s_{n}^{(i)} \mid \mathbf{r}\right)$, for the improved BDFE system with BPSK modulation as

$$
\begin{array}{r}
\Lambda\left(s_{n}^{(i)} \mid \mathbf{r}\right)=\underbrace{\ln \frac{P\left(s_{n}=+1\right)}{P\left(s_{n}=-1\right)}}_{L\left(s_{n}^{(i-1)}\right)}- \\
\underbrace{\sum_{m=1}^{n} \frac{1}{\sigma_{m}^{2}}\left[\left|\rho_{n, m}\left(s_{n}=+1\right)\right|^{2}-\left|\rho_{n, m}\left(s_{n}=-1\right)\right|^{2}\right]}_{L_{E}\left(s_{n}^{(i)}\right)} .
\end{array}
$$

It should be noted the terms related to $\rho_{n, m}\left(s_{n}\right)$, for $m>n$ is independent of $s_{n}$, and they are canceled out during the LLR calculation. Hard decisions of the $n$th bit are obtained through $\operatorname{LLR} \Lambda\left(s_{n}^{(i)} \mid \mathbf{r}\right)$

$$
\hat{s}_{n}^{(i)}=\underset{s \in\{-1,+1\}}{\operatorname{argmax}} \Lambda\left(s_{n}^{(i)} \mid \mathbf{r}\right),
$$

and the extrinsic information $L_{E}\left(s_{n}^{(i)}\right)$ is the soft-output of the SISO equalizer.

As in (20), the extrinsic information of symbol $s_{n}$ is computed by collecting information from a sequence of samples, $\mathbf{r}$. This sequence-based detection is made possible by adopting hard decisions from previous iterations, $\hat{s}_{m}^{(i-1)}$, for $m=1, \cdots, n-1$. These hard decisions can be viewed as the hard a priori information at the input of the equalizer. Therefore, both hard a priori information, $\hat{s}_{n}^{(i-1)}$, and soft $a$ priori information, $L\left(s_{n}^{(i-1)}\right)$, are used during this equalization process.

If the intermediate hard decisions from decision feedback or previous iterations are all correct, then the a posteriori LLR defined in (20) is similar to that described in (9), and the extrinsic information sent out by the improved BDFE is near optimum. In reality, there are usually errors in the intermediate hard decision symbols, especially during the first few iterations. As a result, the LLR calculated in the improved BDFE algorithm is an approximation of the optimum LLR of the MAP algorithm. With the increase of number of iterations, the number of error symbols in the hard decisions keeps decreasing. Therefore, the extrinsic information at the output of the equalizer tends to their optimum values given enough number of iterations. Simulation results show that the algorithm usually converge at five iterations.

Comparing the extrinsic information given in (20) with that defined in (13) for conventional SISO BDFE, we can see that $L_{E}\left(s_{n}^{(i)}\right)$ in the new algorithm is calculated by considering all the received samples related to the information symbol $s_{n}$, and this leads to extra metrics in the decision process. The extra metrics $\rho_{n, m}\left(s_{n}\right)$, for $m>n$, are calculated by using hard a priori information, and this information is discarded by conventional SISO BDFE algorithms. Consequently, the conventional BDFE algorithm can never achieve optimum extrinsic information at the output of the equalizer, even if the hard decisions from decision feedback are all correct.

The computation complexity of the improved BDFE algorithm can be reduced by utilizing the structure of the equivalent channel matrix $\mathbf{G}$. The upper triangular matrix $\mathbf{G}$ is obtained from Cholesky decomposition, and the power of 


$$
\rho_{n, m}\left(s_{n}\right)= \begin{cases}r_{m}-\sum_{k=m}^{N} g_{m, k} \hat{s}_{k}^{(i)}, & n<m \leq N \\ r_{n}-g_{n, n} s_{n}-\sum_{k=n+1}^{N} g_{n, k} \hat{s}_{k}^{(i)}, & m=n \\ r_{m}-g_{m, n} s_{n}-\sum_{k=m}^{n-1} g_{m, k} \hat{s}_{k}^{(i-1)}-\sum_{k=n+1}^{N} g_{m, k} \hat{s}_{k}^{(i)}, & 1 \leq m<n\end{cases}
$$

the elements on its main diagonal is higher than that of the off-diagonal elements. Define the average power of the $l$ th equivalent channel tap as

$$
\bar{p}_{l}=\frac{1}{N-l} \sum_{n=1}^{N-l}\left|g_{n, n+l}\right|^{2}, \text { for } l=0,1, \cdots, N .
$$

The power delay profiles (PDP) of the TU profile and it's minimum phase equivalent are shown in Fig. 2. It's apparent from the figures that the equivalent channel is in its minimum phase form, and the power of the zero-th channel tap (diagonal elements of $\mathbf{G}$ ) is significantly larger than power of the remaining channel taps (off diagonal elements of $\mathbf{G}$ ). Therefore, we can simplify the LLR calculation by discarding channel taps with negligible power without apparently affecting system performance.

Simulations show that neglecting channel taps with power at least $15 \mathrm{~dB}$ below $\bar{p}_{0}$ renders almost the same performance as considering all the channel taps. Therefore, from Fig. 2, only two equivalent channel taps are needed for the LLR calculation for TU profile. The extrinsic information calculation for TU profile can thus be simplified to

$$
\begin{aligned}
L_{E}\left(s_{n}^{(i)}\right) & =-\frac{1}{\sigma_{n}^{2}}\left[\left|\rho_{n, n}\left(s_{n}=+1\right)\right|^{2}-\left|\rho_{n, n}\left(s_{n}=-1\right)\right|^{2}\right] \\
& -\frac{1}{\sigma_{n-1}^{2}}\left[\left|\rho_{n, n-1}\left(s_{n}=+1\right)\right|^{2}-\left|\rho_{n, n-1}\left(s_{n}=-1\right)\right|^{2}\right] .
\end{aligned}
$$

\section{Improved BDFE in M-ary Modulation}

The operations of the improved SISO BDFE for high level modulations is discussed in this subsection.

Define two sets:

$\mathcal{S}_{k}^{(+1)} \triangleq\left\{s_{n} \mid s_{n} \in \mathcal{S}: x_{n, k}=+1\right\}$, for $k=1, \cdots, \log _{2} M$, $\mathcal{S}_{k}^{(-1)} \triangleq\left\{s_{n} \mid s_{n} \in \mathcal{S}: x_{n, k}=-1\right\}$, for $k=1, \cdots, \log _{2} M$,

where $x_{n, k}$ is the $k$ th bit of the binary vector $\mathbf{x}_{n}$ mapped to the symbol $s_{n}$. It's clear that $\mathcal{S}_{k}^{(+1)}$ and $\mathcal{S}_{k}^{(-1)}$ are disjoint and their union is $\mathcal{S}$.

The APP of the bit $x_{n, k}$ in a system with $M$-ary modulation during the $i$ th iteration can be expressed as

$$
\begin{aligned}
P\left(x_{n, k}^{(i)}=+1 \mid \mathbf{r}\right) & =\sum_{s_{n} \in \mathcal{S}_{k}^{(+1)}} P\left(s_{n} \mid \mathbf{r}\right), \\
& =\sum_{s_{n} \in \mathcal{S}_{k}^{(+1)}} \frac{p\left(\mathbf{r} \mid \mathbf{s}_{n}^{(i)}\right) P\left(s_{n}\right)}{p(\mathbf{r})} .
\end{aligned}
$$

where $P\left(s_{n}\right)$ is the a priori probability for the modulation symbol $s_{n}$. If we assume that the $M$-ary modulated symbol $s_{n} \in \mathcal{S}$ is mapped to the binary vector $\mathbf{b}=\left[b_{1}, \cdots, b_{K}\right] \in$

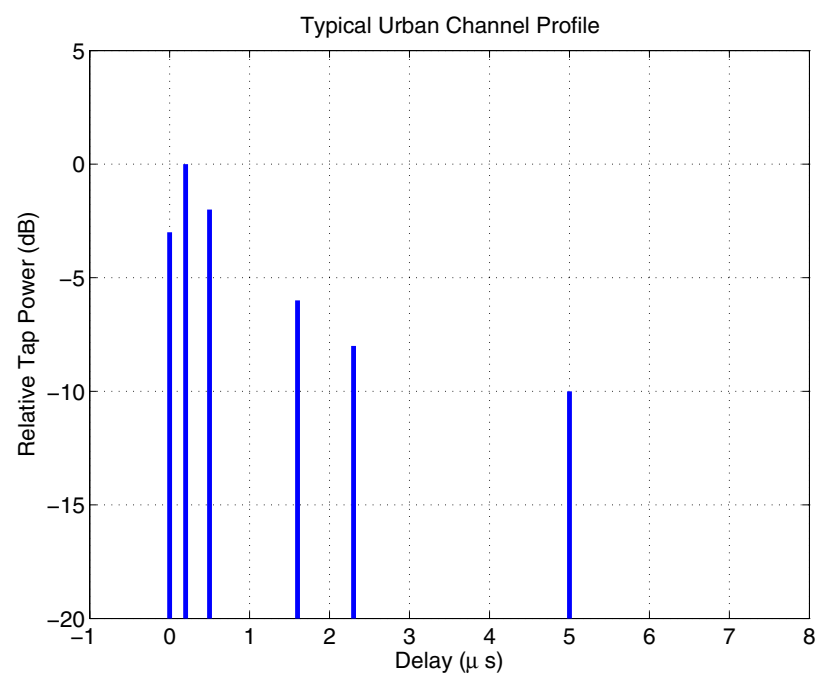

(a) PDP of TU

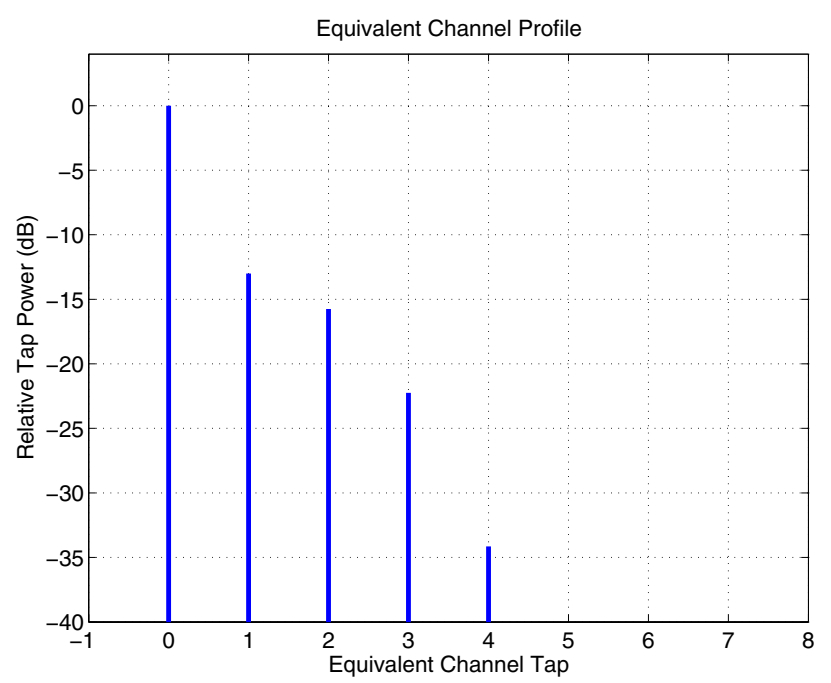

(b) PDP of Equivalent Minimum Phase System

Fig. 2. Power delay profile.

$\mathcal{B}^{1 \times K}$, with $K=\log _{2} M$, then the symbol a priori probability, $P\left(s_{n}\right)$, can be calculated from bit a priori probability, $P\left(x_{n, k}=b_{k}\right)$, as

$$
P\left(s_{n}\right)=\prod_{k=1}^{K} P\left(x_{n, k}=b_{k}\right), \text { for } b_{k} \in\{-1,+1\},
$$

The bit a priori probability can be derived from the bit a priori LLR as

$$
\begin{aligned}
P\left(x_{n, k}=-1\right) & =\frac{1}{1+\exp \left[L\left(x_{n, k}\right)\right]}, \\
P\left(x_{n, k}=+1\right) & =1-P\left(x_{n, k}=-1\right) .
\end{aligned}
$$

The APP $P\left(x_{n, k}^{(i)}=-1 \mid \mathbf{r}\right)$ is defined in a similar manner as (24). 
Combining (9), (18), and (24) leads to the LLR expression of the bit $x_{n, k}$ as

$$
\begin{array}{r}
\Lambda\left(x_{n, k}^{(i)} \mid \mathbf{r}\right)= \\
\ln \frac{\sum_{s_{n} \in \mathcal{S}_{k}^{(+1)}} \exp \left[-\sum_{m=1}^{n} \frac{1}{\sigma_{m}^{2}}\left|\rho_{n, m}\left(s_{n}\right)\right|^{2}\right] P\left(s_{n}\right)}{\sum_{s_{n} \in \mathcal{S}_{k}^{(-1)}} \exp \left[-\sum_{m=1}^{n} \frac{1}{\sigma_{m}^{2}}\left|\rho_{n, m}\left(s_{n}\right)\right|^{2}\right] P\left(s_{n}\right)} .
\end{array}
$$

The a posteriori bit LLR is hard decoded to $\hat{x}_{n, k}^{(i)} \in\{-1,+1\}$, which are then mapped to modulation symbols $\hat{s}_{n}^{(i)} \in \mathcal{S}$ for decision feedback or hard a priori information for the next iteration.

The extrinsic information for bit $x_{n, k}$ at the output of the equalizer during the $i$ th iteration is calculated as

$$
L_{E}\left(x_{n, k}^{(i)}\right)=\Lambda\left(x_{n, k}^{(i)} \mid \mathbf{r}\right)-L\left(x_{n, k}^{(i-1}\right) .
$$

The improved SISO BDFE algorithm is summarized in Table 1.

Table 1. Turbo Equalization Using Improved SISO BDFE

\section{Initialization:}

i. Obtain the equivalent minimum phase system

$$
\mathbf{r}=\mathbf{G s}+\mathbf{v} \text {. }
$$

ii. Soft a priori information: $L\left(x_{n, k}^{(0)}\right)=0$, for $n=1, \cdots, N, k=1, \cdots, M$.

ii. Hard a priori information: $\hat{s}_{n}^{(0)}=0$, for $n=1, \cdots, N$.

Iterations: for the $i$ th iteration

i. Compute $P\left(s_{n}\right)$ by using $L\left(x_{n, k}^{(i-1)}\right)$ and (25) - (27).

ii. Compute $\Lambda\left(x_{n, k}^{(i)}\right)$ based on (19) and (28).

iii. Make hard decision, $\hat{x}_{n, k}^{(i)}$, based on $\Lambda\left(x_{n, k}^{(i)}\right)$. iv. Map $\left[\hat{x}_{n, 1}^{(i)}, \cdots, \hat{x}_{n, K}^{(i)}\right]$ to $\hat{s}_{n}^{(i)}$.

v. Save $\hat{s}_{n}^{(i)}$ in memory.

vi. Compute $L_{E}\left(x_{n, k}^{(i)}\right)$ by using (29).

vii. Deliver $L_{E}\left(x_{n, k}^{(i)}\right)$ to channel decoder.

viii. Feedback $\hat{s}_{n}^{(i)}$ for ISI cancellation.

\section{Complexity Analysis}

The computational complexities of the improved SISO BDFE algorithm and conventional SISO BDFE algorithm are analyzed and compared in this subsection. During the analysis, each complex operation is referred to as one complex multiplication or complex division. Summations or subtractions are not counted since their complexities are much lower compared to multiplications and divisions.

As described in (13), (20), and (28), the computation of the a posteriori LLR for SISO BDFE relies on the metric $\left|\rho_{n, m}\right|^{2}$. It can be seen from (19) that the calculation of each $\left|\rho_{n, m}\right|^{2}$, for $m \leq n$, takes approximately $N-m+2$ complex operations.

For the conventional SISO BDFE algorithm, the calculation of LLR for one bit in one iteration requires the calculation of $M$ metrics, $\left|\rho_{n, n}\left(s_{n}\right)\right|^{2}$, for $s_{n} \in \mathcal{S}$, with $M$ being the modulation constellation size. Therefore, the computational complexity for a block with $N$ symbols in one iteration is in the order of: $\sum_{n=1}^{N} M(N-n+2) \log _{2} M+O(1)=$ $M \frac{N^{2}+3 N}{2} \log _{2} M+O(1)=O\left(\frac{M \log _{2} M}{2} N^{2}\right)$ complex operations, where $O(1)$ is the operations that are independent of $N$ and $M$.

For the improved SISO BDFE algorithm, the detection of each bit LLR requires the calculation of $n M$ metrics, $\left|\rho_{n, m}\left(s_{n}\right)\right|^{2}$, for $m=1, \cdots, n$, and $s_{n} \in \mathcal{S}$. For a block with $N$ symbols, the computational complexity in one iteration is in the order of: $M \sum_{n=1}^{N} \sum_{m=1}^{n}(N-m+2) \log _{2} M+O(1)=$ $M \frac{N^{3}+3 N^{2}+2 N}{3} \log _{2} M+O(1)=O\left(\frac{M \log _{2} M}{3} N^{3}\right)$.

The improved SISO BDFE algorithm can be simplified by neglecting equivalent channel taps with power below a certain threshold, i.e., $P\left(x_{n}^{(i)} \mid \mathbf{r}\right) \approx P\left(x_{n}^{(i)} \mid r_{n}, \cdots, r_{n+J-1}\right)$, where $J<<N$ is the number of effective channel taps. As a result, each bit LLR requires the calculation of $J M$ metrics. For the simplified algorithm, the computational complexity for an $N$-symbol block in one iteration is in the order of $M \sum_{n=1}^{N} \sum_{m=n-J+1}^{n}(N-m+2) \log _{2} M+O(1)=$ $J M \frac{N^{2}+N(J+2)}{2} \log _{2} M+O(1)=O\left(\frac{J M \log _{2} M}{2} N^{2}\right)$.

All the algorithms mentioned above requires the operations of matrix inversion and Cholesky decomposition to formulate the equivalent system as described in (4). For a block with $N$ symbols, the computational complexities of both matrix inversion and Cholesky decomposition are in the order of $O\left(N^{3}\right)$. Therefore, the computational burden of the SISO BDFE algorithms mainly arises from matrix inversion and Cholesky decomposition. Compared to the complexity required for equivalent system formulation, the complexity increase due to the calculation of the $J-1$ extra metrics in the improved SISO BDFE is negligible.

Based on the analysis above, we conclude that the overall computational complexity of the improved SISO BDFE algorithm is on the same order as that of the conventional SISO BDFE algorithm. In addition, the complexity of BDFE algorithms is far below that of the optimum MAP or SOVA equalization algorithms.

\section{Simulation Results}

Simulation results are presented in this section to verify the performance of the proposed algorithm. The convolutional code with rate $1 / 2$ and constraint length 5 is used in the simulations. The octal notation of the generator polynomials is $G=[23,35]_{8}$. The transmitted binary data are divided into length 2048-bit blocks. One block is further cut into subblocks with length 128 bits. BDFE is performed over subblocks, while the convolutional decoding is performed over one block. The frequency selective fading is generated by following the Typical Urban PDP as described in Fig. 2. The channel is constant within one block and varies from block to block. The total power of the channel is normalized to unity.

Fig. 3 investigates the effects of the number of equivalent channel taps, $J$, used by the improved SISO BDFE algorithm, on system performance. The system is 8PSK modulated, and $E_{b} / N_{0}=5 \mathrm{~dB}$. The BER of all iterations shown in the figure decrease monotonically when $J$ increases. $J=$ 1 corresponds to conventional SISO BDFE algorithm. The 
largest performance improvement occurs at the transition from $J=1$ to $J=2$. No apparent performance improvement can be observed for $J \geq 3$. Even though the performance with $J=3$ is slightly better than that of $J=2$, two equivalent taps are used in the remaining simulations for best performance-complexity trade-off. Comparing the results in Fig. 3 with the equivalent PDP shown in Fig. 2, we conclude that channel taps with power $15 \mathrm{~dB}$ below the dominant channel tap are negligible, and thus they can be discarded during the equalization process.

Fig. 4 compares the performance of the proposed algorithm with conventional SISO BDFE algorithm in QPSK modulated system. These two algorithms have the same performance in the first iteration since no a priori information is available to either of the algorithms. The proposed algorithm outperforms the conventional BDFE algorithm starting from iteration 2, thanks to the extra hard a priori information utilized by the new algorithm. From the figure, the BER of the conventional BDFE algorithm after its 5th iteration is only comparable to the 2 nd iteration results of the improved algorithm. The results obtained from the optimum MAP equalizer is also plotted in the figure. It can be seen that at BER level $3 \times 10^{-4}$ and after 5 iterations, the proposed algorithm is $0.5 \mathrm{~dB}$ better than the conventional BDFE algorithm, and $0.5 \mathrm{~dB}$ away from the optimum MAP equalizer.

The performance of the improved BDFE algorithm is further compared with that of the SISO MMSE algorithm described in [9] as shown in Fig. 5. The simulation is performed in 8PSK modulated system. The SISO MMSE algorithm only uses the soft a priori information, whereas both soft and hard a priori information are used by the improved BDFE algorithm. We have the following two observations from the figure. First, as expected, the non-linear BDFE algorithm outperforms the linear MMSE algorithm in the first iteration, because of the help from decision feedback. Second, the proposed algorithm converges much faster compared to the SISO MMSE algorithm. For example, the 10th iteration results of the MMSE algorithm is similar to the 2nd iteration results of the improved BDFE algorithm. Since the computational complexity of soft MMSE algorithm in one iteration is comparable to that of the improved BDFE algorithm, much more computation is going to be required by the soft MMSE algorithm to achieve the same performance as the improved BDFE algorithm. This fast convergence property of the proposed algorithm is contributed by the combination of hard a priori information and soft $a$ priori information.

\section{CONCLUSION}

An improved SISO BDFE for low complexity turbo equalization was proposed in this paper. The new algorithm performs equalization by using the combination of soft a priori information from trellis-based channel decoder and hard $a$ priori information from BDFE in previous iteration. The adoption of hard a priori information enables sequence-based detection, which results in more accurate a posteriori LLR at the output of the equalizer. The computational complexity of the improved BDFE algorithm is further reduced by discarding negligible channel taps of the equivalent minimum phase system. It was concluded from analysis that the complexity

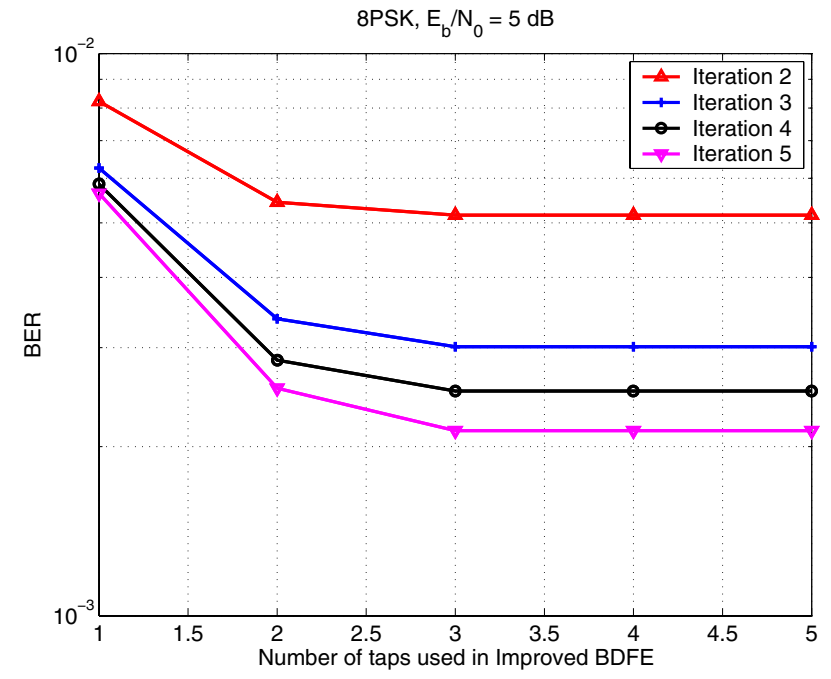

Fig. 3. Effects of number of equivalent channel taps on performance of the improved BDFE algorithm.

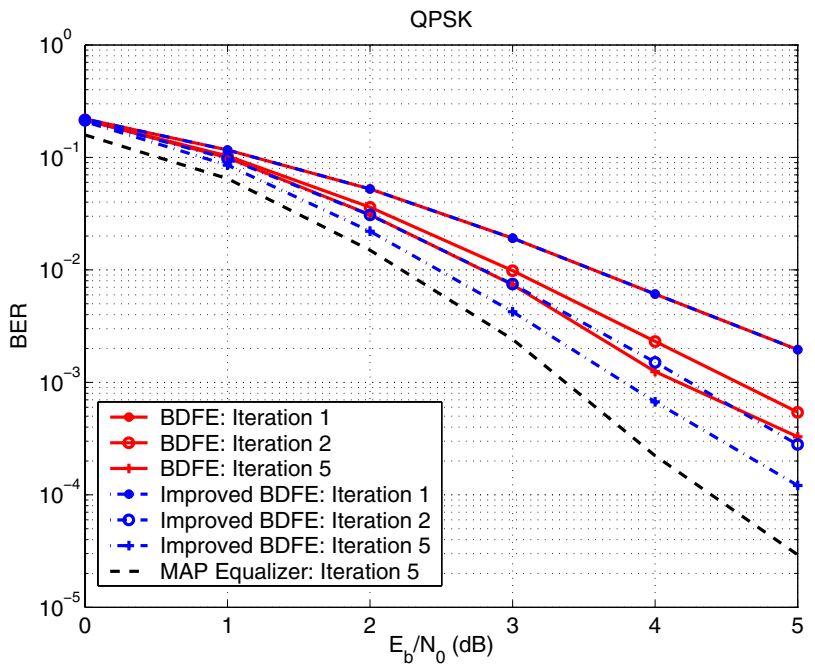

Fig. 4. BER performance in QPSK modulated system.

of the proposed algorithm is on the same order at that of conventional SISO BDFE algorithm. Simulation results showed that the proposed algorithm outperforms conventional SISO BDFE algorithm and linear SISO MMSE algorithm. In addition, due to the adoption of hard a priori information, the proposed algorithm converges much faster compared to algorithms which only utilize soft a priori information.

\section{ACKNOWLEDGMENTS}

The authors are grateful to the anonymous reviewers and the Editor, Dr. Zhi Tian, for their careful reviews and valuable comments that have helped us improve the quality of the paper.

\section{REFERENCES}

[1] C. Douillard, et al., "Iterative correction of intersymbol interference: Turbo-equalization,” Euro Trans. Telecommun., vol. 6, no. 5, pp. 507511, Sep.-Oct. 1995.

[2] J. Hagenauer, "A Viterbi algorithm with soft-decision outputs and its application," in Proc. IEEE GLOBECOM, Nov. 1989, pp. 1680-1686. 


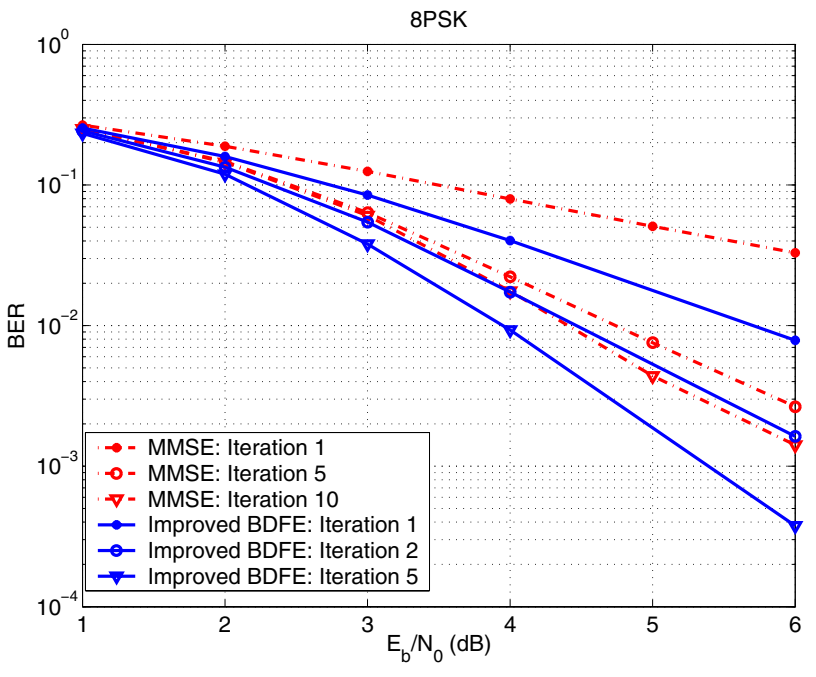

Fig. 5. BER performance in 8PSK modulated system.

[3] J. Hagenauer and L. Papke, "Decoding 'Turbo'-codes with the soft output Viterbi algorithm," in Proc. IEEE Int. Symp. Inform. Theory, July 1994, pp. 164.

[4] G. Bauch and V. Franz, "A comparison of soft-in/soft-out algorithms for 'Turbo detection'," in Proc. Int. Conf. Telecomm., June 1998, pp. 259-263.

[5] L. R. Bahl, J. Cocke, F. Jelinek, and J. Raviv, "Optimal decoding of linear codes for minimizing symbol error rate," IEEE Trans. Inform. Theory, vol. 20, pp. 284-287, Mar. 1974.

[6] C. Berrou, A. Glavieux, and P. Thitimajshima, "Near Shannon limit error-correcting and decoding: Turbo codes (1)," in Proc. IEEE Int. Conf. Commun., May, 1993, vol. 2, pp. 1064-1070.

[7] X. Wang and H. V. Poor, "Iterative (Turbo) soft interference cancellation and decoding for coded CDMA," IEEE Trans. Commun., vol. 47, pp. 1046-1061, July 1999.

[8] M. Tuchler, A. C. Singer, and R. Koetter, "Minimum mean squared error equalization using a priori information," IEEE Trans. Signal Processing, vol. 50, pp. 673-683, Mar. 2002.

[9] M. Tuchler, R. Koetter and A. C. Singer, "Turbo equalization: Principles and new results," IEEE Trans. Commun., vol. 50, pp. 754-767, May 2002.

[10] A. Dejonghe and L. Vandendorpe, "Turbo-equalization for multilevel modulation: An efficient low complexity scheme," in Proc. IEEE Int. Conf. Commun., May. 2002, pp. 1863-1867.

[11] S. L. Ariyavisitakul and Y. Li, "Joint coding and decision feedback equalization for broadband wireless channels," IEEE J. Select. Areas Commun., vol. 16, pp. 1670-1678, Dec. 1998.

[12] C. Berrou, P. Adde, E. Angui, and S. Faudeil, "A low complexity softoutput Viterbi decoder architecture," in Proc. IEEE Int. Conf. Commun., May 1993, pp. 737-740.

[13] P. Strauch, et al., "Turbo equalization for an 8-PSK modulation scheme in a mobile TDMA communication system," in Proc. IEEE Veh. Technol. Conf., Sep. 1999, vol. 3, pp. 1605-1609.

[14] M. J. Lopez, K. Zangi, and J. Cheng, "Reduced-complexity MAP equalizer for dispersive channels," in Proc. IEEE Veh. Technol. Conf., Sep. 2000, pp. 1371-1375.

[15] A. Berthet, R. Visoz, and P. Tortelier, "Sub-optimal turbo-detection for coded 8-PSK signals over ISI channels with application to EDGE advanced mobile system," in Proc. IEEE PIMRC, Sep. 2000, pp. 151157.

[16] W. H. Gerstacker, R. R. Muller, and J. B. Huber, "Iterative equalization with adaptive soft feedback," IEEE Trans. Commun., vol. 48, pp. 14621466, Sep. 2000.

[17] X. Tang and Z. Ding, "Turbo equalization for EDGE system with DDFSOVA," in Proc. 35th Asilomar Conf. Signals, Systems Computers, Nov. 2001, pp. 295-299.

[18] R. Otnes and M. Tuchler, "Low-complexity turbo equalization for timevarying channels," in Proc. IEEE Veh. Technol. Conf., May 2002, pp. 140-144.

[19] C. Xiao, J. Wu, S. Y. Leong, Y. R. Zheng, and K. B. Letaief, "A discrete- time model for triply selective MIMO Rayleigh fading channels," IEEE Trans. Wireless Commun., vol. 3, pp. 1678-1688, Sep. 2004.

[20] ETSI. GSM 05.05, "Radio transmission and reception," ETSI EN 300 910 V8.5.1, Nov. 2000.

[21] A. Stamoulis, G. B. Giannakis, and A. Scaglione, "Block FIR decisionfeedback equalizers for filterbank precoded transmissions with blind channel estimation capabilities," IEEE Trans. Commun., vol. 49, pp. 69-83, Jan. 2001.

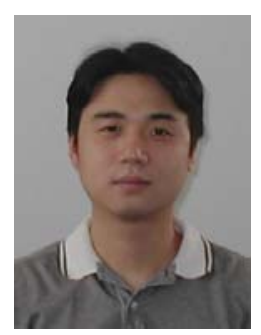

Jingxian Wu (S'02-M'06) received the B.S. (EE) degree from Beijing University of Aeronautics and Astronautics, Beijing, China, in 1998, the M.S. (EE) degree from Tsinghua University, Beijing, China, in 2001, and the Ph.D. (EE) degree from the University of Missouri at Columbia, MO, USA, in 2005.

Dr. Wu is an Assistant Professor at the Department of Engineering Science, Sonoma State University, Rohnert Park, CA, USA. His research interests mainly focus on the physical layer of wireless communication systems, including multicarrier communications, error performance analysis, space-time coding, channel estimation and equalization, and spread spectrum communications.

Dr. Wu is currently an Associate Editor for the IEEE TRANSACTIONS on Vehicular Technology. He has served as a Technical Program Committee member for a number of international conferences, including the IEEE Global Telecommunications Conference, the IEEE Wireless Communications and Networking Conference, and the IEEE International Conference on Communications. Dr. Wu is a member of Tau Beta Pi.

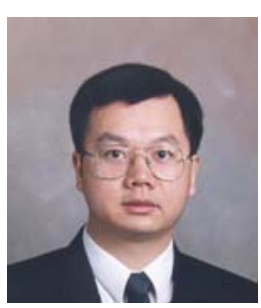

Chengshan Xiao (M'99-SM'02) received the B.S. degree from the University of Electronic Science and Technology of China, Chengdu, China, in 1987, the M.S. degree from Tsinghua University, Beijing, China, in 1989, and the Ph.D. degree from the University of Sydney, Sydney, Australia, in 1997, all in electrical engineering.

From 1989 to 1993, he was on the Research Staff and then became a Lecturer with the Department of Electronic Engineering at Tsinghua University, Beijing, China. From 1997 to 1999, he was a Senior Member of Scientific Staff at Nortel Networks, Ottawa, ON, Canada. From 2000 to 2007, he was an Assistant Professor and then became an Associate Professor at the University of Missouri-Columbia, Columbia, MO. He is now an Associate Professor with the Department of Electrical and Computer Engineering at the University of Missouri-Rolla, Rolla, MO. His research interests include wireless communications and signal processing. He has published extensively in these areas. He holds three U.S. patents. His algorithms have been implemented into Nortel's base station radios with successful technical field trials and network integration.

Dr. Xiao is the founding Area Editor for Transmission Technology of the IEEE Transactions on Wireless Communications. Previously, he was an Associate Editor for the IEEE TRANSACTIONS ON VEHICULAR Technology, the IEEE TRAnsactions on Circuits and Systems$\mathrm{I}$, and the International Journal of Multidimensional Systems and Signal Processing. He served as a Technical Program Co-Chair for the 2007 IEEE Wireless Communications and Networking Conference, and Co- Chair for the 2008 IEEE ICC Wireless Communications Symposium. He is the founding Chair of the IEEE Communications Society Technical Committee on Wireless Communications and a member of the IEEE Communications Society Technical Activity Council.

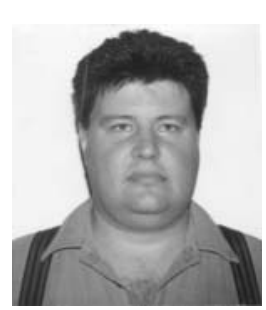

Jan C. Olivier received the B.Eng (Electronics), M.Eng (Electronics) and Ph.D. (Electronics) summa cum laude from the University of Pretoria in 1985, 1986, and 1990 respectively. He joined Northwestern University, R.S.A. in 1991, then Daimler Benz Aerospace, Ottawa, Canada in 1995, Bell Northern Research (BNR), Ottawa, Canada in 1997, and Nokia Research Center, Dallas, TX, U.S.A. in 1999 as a Principle Scientist. In May 2003, he was appointed as a Professor of Electrical Engineering at the University of Pretoria, where he is currently also the Director of the Telkom Centre of Excellence in Telecommunications Engineering (Ceteis). He serves as an Associate Editor for the IEEE Transaction on Wireless Communications, and his research interests are in estimation, detection and computational methods. 